\title{
Elliptic behaviour in the sawtooth standard map
}

\author{
Peter Ashwin \\ Department of Mathematical and Computing Sciences \\ University of Surrey \\ Guildford GU2 5XH \\ U.K.
}

June 4, 1997

\begin{abstract}
This paper examines the standard map with sawtooth nonlinearity when the eigenvalues of the Jacobian lie on the unit circle. This is an area-preserving map of the torus to itself that is linear except on a line on which it is discontinuous. We discuss the closure of the set of images of the discontinuity and present numerical evidence that its Lebesgue measure is positive. Moreover, we present evidence that the measure of the closure of images of the discontinuity changes continuously with the parameter $k$. This means that the sawtooth standard map may exhibit coexistence of two positive measure subsets on which the dynamics is respectively regular and irregular in a certain sense.

In the appendix we show that this map is equivalent to a map studied by electronics engineers as a model for the quiescent behaviour of a linear lossless digital filter with "two's complement" overflow.
\end{abstract}

\section{Introduction}

The standard map with sawtooth nonlinearity is defined by

$$
\left(\begin{array}{l}
x^{\prime} \\
y^{\prime}
\end{array}\right)=f\left(\begin{array}{l}
x \\
y
\end{array}\right)=\left(\begin{array}{c}
x+k \operatorname{saw}(y) \\
y+x+k \operatorname{saw}(y)
\end{array}\right)
$$

for $(x, y) \in[0,1)^{2}$, where

$$
\operatorname{saw}(y)=r(y)-\frac{1}{2}
$$

and the remainder function $r(y)$ is defined by

$$
\begin{array}{ll}
r(y)=y & y \in[0,1) \\
r(y+1)=r(y) & y \in \mathbf{R}
\end{array}
$$


i.e. $r(y)=y-\lfloor y\rfloor$. This map and variants of it have been studied by several authors as a linear model of the Chirikov-Taylor standard map

$$
\left(\begin{array}{c}
x^{\prime} \\
y^{\prime}
\end{array}\right)=\left(\begin{array}{c}
x+\frac{k}{2 \pi} \sin 2 \pi y \\
y+x+\frac{k}{2 \pi} \sin 2 \pi y
\end{array}\right)
$$

obtained by linearising the map about its fixed point at $\left(0, \frac{1}{2}\right)$. The map (1) has the following properties

1. It is a smooth map on a full measure subset of the phase space.

2. It preserves area (more precisely, two dimensional Lebesgue measure is invariant) and moreover the map has constant Jacobian

$$
D f=\left(\begin{array}{cc}
1 & k \\
1 & 1+k
\end{array}\right) .
$$

Therefore all trajectories that do not hit a discontinuity have Lyapunov exponents equal to the real parts of the eigenvalues of this matrix.

Because of constancy of the Jacobian, the Lyapunov exponents are simply the logarithms of the eigenvalues and so we can classify the map as follows:

\begin{tabular}{l|c|c} 
Regime & $k$ & Lyapunov exponents \\
\hline elliptic & $-4<k<0$ & 0 \\
hyperbolic & $k<-4$ or $k>0$ & $1+\frac{k}{2} \pm \sqrt{k\left(1-\frac{k}{4}\right)}$ \\
parabolic & $k=-4$ or $k=0$ & 0 (nilpotent Jacobian)
\end{tabular}

Many studies of map have focussed on the hyperbolic regime; in particular Percival [9] has shown that it is possible to get a good description of cantori of the hyperbolic map by looking at orbits minimising the associated variational problem. This line of investigation has been further pursued [10,3] to examine flux and resonance.

Wojtkowski [11] has looked at a map of the form (1) where the function $\operatorname{saw}(y)$ is replaced by a continuous piecewise linear function. In particular, he has shown that both elliptic and hyperbolic behaviour can coexist in this mapping at some parameter values. We restrict to the simpler problem where all orbits are elliptic.

A mathematically similar problem concerns the dynamics of a second order linear filter realised using arithmetic that has a 'periodic' overflow. This is called the "two's complement" overflow oscillation problem and has been studied by many authors in the electronics engineering community, see e.g. [2, 4, 5, 8].

We show in the appendix that the map (1) is equivalent to the lossless case of the overflow oscillation problem under a piecewise linear change of coordinates. In particular, any property of the sawtooth standard map also holds for a lossless overflow oscillation system, and vice versa. Some of the results in the next sections have been shown for the latter problem; the equivalence then shows simply that they apply to the sawtooth map. 


\section{The elliptic regime; general considerations}

From now on, we assume that the map is in the elliptic regime, i.e. $-4<k<0$ so we can find a unique $\theta \in(0, \pi)$ such that

$$
2 \cos \theta=2+k
$$

We use the parameters $\theta$ and $k$ interchangeably, assuming they are related by the above formula; at times we refer to $k(\theta)$ to make this relationship explicit.

We consider the sawtooth map $f: I \rightarrow I$ defined by (1) with $I=[0,1)^{2}$; the map is linear and continuous except on the discontinuity (with respect to the topology of the torus $\mathbf{R}^{2} / \mathbf{Z}^{2}$ ) at

$$
\mathcal{D}=\{(x, y) \in I: y=0\} .
$$

This set has two dimensional Lebesgue measure zero. We can perform a local change of coordinates to

$$
\left(\begin{array}{l}
X \\
Y
\end{array}\right)=M\left(\begin{array}{l}
x \\
y
\end{array}\right)=\left(\begin{array}{cc}
\cos \theta & \sin \theta \\
0 & 1
\end{array}\right)\left(\begin{array}{l}
x \\
y
\end{array}\right)
$$

which makes the map locally equivalent to a rigid rotation through an angle $\theta$.

Define the set of preimages and images of the discontinuity $\mathcal{D}^{ \pm}=\cup_{k \in \mathbf{Z}} f^{n}(\mathcal{D})$ and a full measure subset of the phase space

$$
\tilde{I}=I \backslash \mathcal{D}^{ \pm}
$$

The restricted map $f: \tilde{I} \rightarrow \tilde{I}$ is invertible and its dynamics are independent of what values are taken on the discontinuity. There is a natural splitting of phase space into a disjoint union of two measurable invariant subsets, namely

$$
D=\overline{\mathcal{D}^{ \pm}} \backslash \mathcal{D}^{ \pm}, \quad D^{c}=\tilde{I} \backslash D .
$$

These sets can be characterised in the following way:

$D$ consists of all points whose iterates approach the discontinuity arbitrarily closely (but never land on it).

$D^{c}$ consists of all points whose iterates remain a bounded distance away from the discontinuity.

Note that the above decomposition can also be formed in the hyperbolic case, in which case $D^{c}$ is empty and $D$ has full measure. We define

$$
\Phi(k)=\ell(D)
$$

with $\ell(\cdot)$ denoting two dimensional Lebesgue measure. The dynamics on $D^{c}$ are easy to understand in the light of the following result, see e.g. $[4,1]$.

Lemma $1 D^{c}$ consists of a union of open convex sets. These sets are ellipses if $\theta / \pi \in \mathbf{R} \backslash \mathbf{Q}$ and polygons if $\theta / \pi \in \mathbf{Q}$. 
ProOF This follows on examining the construction of $\mathcal{D}^{ \pm}$; at each stage we add an extra line to the previous set; this will subdivide convex polygons into convex polygons and so the limit will consist of convex polygons. If $\theta / \pi$ is irrational then the boundary will have a dense set of points with tangents in $\mathcal{D}$ and so will be an ellipse.

The dynamics on $D$ may be sensitive even though the Lyapunov exponents are zero; see also the example in [1].

\section{Action of the map on elliptical disks.}

Length preservation The map $f$ on $I$ is locally conjugate to a rigid rotation, with the result that elements in a certain family of ellipses are mapped onto themselves. Consider the quadratic form $x^{T} Q x$ with

$$
Q=\left(\begin{array}{cc}
-\frac{2}{k} & -1 \\
-1 & 2
\end{array}\right)
$$

which is positive definite precisely when $-4<k<0$. This can be used to define a norm by $|\mathbf{x}|_{Q}=\sqrt{\mathbf{x}^{T} Q \mathbf{x}}$. If the line joining $\mathbf{x}_{1}$ and $\mathbf{x}_{2}$ does not intersect the discontinuity of the map then $f$ preserves $|\cdot|_{Q}$, i.e.

$$
\left|\mathbf{x}_{1}-\mathbf{x}_{2}\right|_{Q}=\left|f\left(\mathbf{x}_{1}\right)-f\left(\mathbf{x}_{2}\right)\right|_{Q}
$$

Given a point $\mathbf{x} \in A$ and a radius $r \geq 0$ we define an elliptical disk in $A$

$$
E_{r}(\mathbf{x})=\left\{\mathbf{y} \in A:|\mathbf{y}-\mathbf{x}|_{Q}<r\right\} .
$$

Note that if $E_{r}(\mathbf{x}) \cap \mathcal{D}=\emptyset$ then $f\left(E_{r}(\mathbf{x})\right)=E_{r}(f(\mathbf{x}))$. If we consider the three dimensional manifold of all such disks $\mathcal{E}=\left\{E_{r}(\mathbf{x}): \mathbf{x} \in A\right.$ and $\left.r>0\right\}$ there is a subset $\mathcal{E}_{0} \subset \mathcal{E}$ of disks in $A$ that do not intersect the discontinuity of $F$. We define inductively a nested sequence of subsets $\mathcal{E}_{n+1}=f^{-1}\left(\mathcal{E}_{n}\right) \cap \mathcal{E}_{n}$ with limit $\mathcal{E}_{\infty}=\cap_{n \in \mathbf{Z}} \mathcal{E}_{n}$. There is a partial order on disks $E, F \in \mathcal{E}_{\infty}$ given by $E \preceq F$ if $E \subset F$. We define a cone in $\mathcal{E}$ with vertex $E$ by $\operatorname{Cone}(E)=\{F \in \mathcal{E}: F \preceq E\}$. Note that trivially, the set $\mathcal{E}_{\infty}$ contains all cones with vertices in $\mathcal{E}_{\infty}$. We say $E$ is a maximal disk if it is a maximum under $\preceq$. Cone $(E)$ is called a maximal cone if $E$ is a maximal disk. We define $\mathcal{E}_{\infty}^{\max }$ to be the set of maximal cones in $\mathcal{E}_{\infty}$. Note that $D$ splits $\tilde{I}$ into a disjoint union of connected components, and that $\preceq$ is not defined between disks contained in different connected components.

There is a stronger concept of maximality we can use; consider the equivalence class of disks in $\mathcal{E}_{\infty}$ defined by the relation $E \sim F$ if there is a finite sequence of disks relating $E$ to $F$ under $\preceq$. We say a disk is strongly maximal if all other disks in the equivalence class have strictly smaller diameter and write $\hat{\mathcal{E}}_{\infty}^{\text {max }}$ to denote all such strongly maximal disks. Note that $\hat{\mathcal{E}}_{\infty}^{\max } \subseteq \mathcal{E}_{\infty}^{\max }$. 


\subsection{Decomposition of $\mathcal{E}_{\infty}$ into maximal disks}

We now obtain some elementary results about the dynamical structure $f$ induces on $\mathcal{E}_{\infty}$. In particular we show that maximal disks are periodic. For irrational angles, $\theta / \pi \in \mathbf{R} \backslash \mathbf{Q}$ we show that

$$
\mathcal{E}_{\infty}^{\max }=\hat{\mathcal{E}}_{\infty}^{\max }
$$

i.e. a disk is maximal if and only if it is strongly maximal. This means that we can characterise the dynamics in $D^{c}$ by looking at the set of strongly maximal disks. For rational angles, $\theta / \pi \in \mathbf{Q}$ the inclusion $\mathcal{E}_{\infty}^{\max } \supset \hat{\mathcal{E}}_{\infty}^{\text {max }}$ is strict and there are many maximal disks that are not strongly maximal.

Lemma 2 Any maximal disk is periodic.

Proof Consider a maximal disk; this lies within the closure of a component $C$ of $D^{c}$. If $C$ is a disk then the unique maximal disk is $\bar{C}$. If $C$ is a polygon then all points in $C$ are periodic and so again any maximal disk must be periodic as its centre is.

Theorem 1 If $\theta / \pi \in \mathbf{R} \backslash \mathbf{Q}$ then $\mathcal{E}_{\infty}^{\max }=\hat{\mathcal{E}}_{\infty}^{\max }$.

Proof By Lemma 2, $E \in \mathcal{E}_{\infty}^{\max }$ has a minimal period $n$ such that $f^{n}(E)=E$. If we examine $E_{0}=D \cap \bar{E}$ then (i) $E_{0}$ is non-empty (otherwise $E$ is not maximal), (ii) $E_{0}$ is $f^{n}$ invariant and (iii) $f^{n}$ acts as a rotation by the irrational angle $n \theta$ on $E_{0}$. Therefore $E_{0}$ is dense in $\partial E$. In particular, $F \sim E$ implies that $F \subset E$ which gives the result.

Lemma 3 If $\theta / \pi \in \mathbf{Q}, \theta \neq 0$ then within each equivalence class of $\mathcal{E}_{\infty}$ under $\sim$ there is a unique strongly maximal $E$ but a continuum of maximal $E$.

Proof This follows because any $E$ within such an equivalence class is contained within a convex polygon.

As a result of this we can see that disks in $\hat{\mathcal{E}}_{\infty}^{\max }$ are disjoint and $U^{\max }=\bigcup_{E \in \hat{\mathcal{E}}_{\infty}^{\max }} E$ is strictly contained in $D^{c}$ for $\theta / \pi \in \mathbf{Q}$.

\subsection{Measure of strongly maximal disks}

To approach the main conjectures of this paper, we consider the dependence of the measure of strongly maximal disks on the parameter $\theta$. To this end we define

$$
P(\theta)=1-\ell\left(U^{\max }\right)
$$

and note that by Theorem 1

$$
P(\theta)=\Phi(k(\theta)) \quad \text { for } \quad \theta / \pi \in \mathbf{R} \backslash \mathbf{Q}
$$

However $P(\theta)=0$ for $\theta / \pi \in \mathbf{Q}$. Note that maximal disks are robust and so it is relatively easy to show that $P(\theta)$ is upper semicontinuous for all $\theta$ (although $\Phi(\theta)$ is not). Lower semicontinuity is where the problem lies! 
Lemma $40<P(\theta)<1$ for all $\theta / \pi \in \mathbf{Q}$.

ProOF For rational angles, $D^{c}$ consists of polygons; for each polygon $P$ we have $\ell\left(P \cap \hat{U}^{\mathrm{max}}\right)>0$ and $\ell\left(P \cap\left(I \backslash U^{\max }\right)\right)>0$ and so $\ell(\Phi(\theta))$ is bounded away from 0 and 1.

The next two conjectures are suggested by the numerical experiments outlined in Section 4.

Conjecture $1 P(\theta)$ is non-zero for all $\theta \neq 0$.

Conjecture $2 P(\theta)$ is a continuous function of $\theta \neq 0$.

The first conjecture is a restatement of a conjecture in [2] in the light of the appendix to this paper. The second conjecture implies the first on applying Lemma 4.

Remark 1 One might initially suspect that there are no coverings of the square by a countable number of disjoint circles such that the covering has full measure. This is however not the case; one can explicitly construct a full measure cover. Divide the complement of the first circle into a countable number of squares and then inductively define a countable number of squares within these. Taking the limit one can show that the union of the circles has full measure.

Remark 2 One can also construct dense circle packings that have less than full measure, for example in the following way. Given a sequence $r_{i}$ such that $\pi \sum r_{i}^{2}<1$ and a sequence $\mathbf{x}_{j}$ of points in $(0,1)^{2}$ that are dense in $[0,1]^{2}$ we can construct a dense circle packing by placing disks of radius $r_{i}$ at points $\mathbf{x}_{j}$ (missing out radii or points that would result in overlapping disks). The total Lebesgue measure of the union of disks will then be less than or equal to $\pi \sum r_{i}^{2}$. Moreover, every point will either be in the closure of a disk, or at a limit point of centres of disks.

\section{Numerical approximation of $D$}

In order to test the validity of the above conjectures we have constructed numerical approximations of the set $D$ using box-counting on a discretised phase space. This approximation is similar to that used for the overflow oscillation problem detailed in [2]; we refer the reader to [2] for more details.

The phase space was subdivided into a grid formed of $2^{n}$ by $2^{n}$ intervals. An initial point was chosen at the centre of each of the boxes on one side of the discontinuity $\mathcal{D}$ and its orbit was computed until it returned back to the starting box, at

which point it was deemed to have closed. In this way we get an approximation of the set $D$ by marking each of the boxes visited by a trajectory coming close to $\mathcal{D}$. The measure of the set was approximated by counting the number of boxes hit as a proportion of $2^{2 n}$. This approximation of $\Phi(k)$ is denoted by $\Phi_{n}(k)$.

Assume that the set of boxes is approximately an $\epsilon$-neighbourhood of $D$ where $\epsilon \sim 2^{-n}$. Assume additionally that the measure of the neighbourhood scales like

$$
\ell\left(B_{\epsilon}(D)\right)=\ell(D)+A \epsilon^{2-d}
$$


to leading order in $\epsilon$, where $d$ is the exterior dimension of the set $D[6,7]$ then we may expect to see the scaling

$$
\Phi_{n}(k)=\Phi_{\infty}+B e^{C n}
$$

in the limit $n \rightarrow \infty$. Figure 1 shows that this is indeed the case, where we have taken $k=-0.5, \theta=\cos ^{-1}(0.75)$. The scaling is consistent with $\Phi_{\infty} \sim 0.12$. Figure 2 shows the computed approximation to $D$ where $n=10$. In contrast Figure 3 shows the approximation of $D$ and the convergence of $\Phi_{n}$ to 0 as expected at this rational value of $\theta / \pi=1 / 4(k=-2+\sqrt{2})$.

Figure 4 shows an extrapolated value of $\Phi_{\infty}(k)$ as a function of $k$; this was computed by extrapolating values of $\Phi_{n}$ for $n=6,8,10$ and 12 to the limit $\infty$ using equation (4). Apart from the excursions to zero expected for rational values of $\theta / \pi$, the extrapolated curve remains close to a continuous curve. In particular, near $k=-1$ the curve seems to limit to a value $\Phi(\pi / 2)=0.16867$ that can be explicitly computed.

Figure 5 shows extrapolated values of $\Phi_{\infty}(k)$ using fits to (a) $n=6,8$ and 10 and (b) $n=8,10$ and 12. Note that in the latter case, fewer points are detected as spurious and there are generally fewer outliers to the general continuous trend of the predictions.

\section{Discussion}

We have conjectured that for a full measure set of parameters in the sawtooth standard map, there is coexistence of elliptic islands $D^{c}$ with a connected "chaotic sea" $D$, such that both of these have positive Lebesgue measure. Moreover we have shown that the total area of invariant ellipses inscribed in $D^{c}\left(U^{\max }\right)$ is equal to $D^{c}$ whenever $\theta / \pi \in \mathbf{R} \backslash \mathbf{Q}$. We conjecture that the area of $U^{\max }$ changes continuously with parameter.

These conjectures have been supported by numerical simulations, although the dynamical behaviour in $D$ is presumably very complex [1]; we do not examine this here. Fairly basic questions still remain unanswered; for example, is it true that the set of elliptic islands $\left(D^{c}\right)$ is dense in the phase space?

It is remarkable that the chaotic sea $(D)$ in this simple piecewise linear model has many features of the chaotic sea in smooth area preserving maps, even though in all cases there is a full measure subset whose Lyapunov exponents are zero and so any chaotic behaviour must be non-hyperbolic.

\section{Acknowledgements}

Thanks to T. Bridges, W. Chambers and G. Petkov for very interesting conversations concerning this work. 


\section{References}

[1] P. Ashwin. "Non-smooth invariant circles in digital overflow oscillations". Proceedings of NDES96: fourth international workshop on Nonlinear Dynamics of Electronic Systems, Seville, Spain 1996.

[2] P. Ashwin, W. Chambers and G. Petkov. Lossless digital filter overflow oscillations; approximation of invariant fractals. To appear in Intl. J. Bifn. and Chaos (1997).

[3] Q. Chen and J.D. Meiss. Flux, resonances and the devil's staircase for the sawtooth map. Nonlinearity 2:347-356 (1989).

[4] L.O. Chua and T. Lin. Chaos in digital filters. IEEE Trans. CAS 35:648-658 (1988).

[5] A.C. Davies. Nonlinear oscillations and chaos from digital filter overflow. Phil. Trans. R. Soc. Lond. A 353:85-99 (1995).

[6] J.D. Farmer, Sensitive dependence on parameters in nonlinear dynamics. Phys. Rev. Lett. 55:351-354 (1985).

[7] C. Grebogi, S.W. McDonald, E. Ott \& J.A. Yorke. Exterior dimensions of fat fractals Phys. Lett. A 110:1-4 (1985).

[8] L. Kocarev, C.W. Wu and L.O. Chua. Complex behaviour in Digital filters with overflow nonlinearity: analytical results. IEEE Trans CAS-II 43:234-246 (1996).

[9] I.C. Percival. Variational principles for invariant tori and cantori. Am. Inst. Phys. Conf. Proc. 57:302-310 (1979).

[10] I.C. Percival and F. Vivaldi. A linear code for the sawtooth and the cat maps. Physica D 27:373-386 (1987).

[11] M. Wojtkowski. A model problem with the coexistence of stochastic and integrable behaviour. Commun. Math. Phys. 80:453-464 (1981).

\section{Appendix: equivalence to overflow oscillation prob- lem}

In this section we consider the lossless digital linear filter overflow oscillation problem $[2,5,4,8]$ described by the map

$$
\left(\begin{array}{c}
u^{\prime} \\
v^{\prime}
\end{array}\right)=\left(\begin{array}{c}
v \\
g(-u+a v)
\end{array}\right)
$$


for $(u, v) \in[-1,1)^{2}$, where we have a periodic 'overflow function' (so-called 'two's complement overflow")

$$
g(x)=2 r\left(\frac{x+1}{2}\right)-1
$$

and $a$ is a real constant that determines the feedback. This map arises in the study of second order linear filters with linear part

$$
\left(\begin{array}{cc}
0 & 1 \\
-1 & a
\end{array}\right)
$$

realised using arithmetic that overflows according to a function $g(x)$. This overflow can be thought of as a continuum limit of addition of binary numbers in two's complement notation; hence the name.

We show that this is conjugate to the elliptic sawtooth standard map by the piecewise linear change of coordinates

$$
\left(\begin{array}{l}
u \\
v
\end{array}\right)=\left(\begin{array}{c}
2 r(x-y)-1 \\
1-2 y
\end{array}\right)
$$

mapping $(x, y) \in[0,1]^{2}$ to $(u, v) \in[-1,1]^{2}$. This is almost everywhere invertible with inverse

$$
\left(\begin{array}{l}
x \\
y
\end{array}\right)=\left(\begin{array}{c}
r\left(\frac{u-v}{2}\right) \\
\frac{-v+1}{2}
\end{array}\right) .
$$

The remainder function $r(y)$ has the following properties:

$$
\begin{aligned}
& r(r(y))=r(y) \\
& r(-r(y))=1-r(y)=r(-y) \\
& r(y+z)=r(r(y)+z)
\end{aligned}
$$

and we can use these to transform (1) into

$$
\begin{aligned}
\left(\begin{array}{l}
u^{\prime} \\
v^{\prime}
\end{array}\right) & =\left(\begin{array}{c}
2 r\left(x^{\prime}-y^{\prime}\right)-1 \\
1-2 y^{\prime}
\end{array}\right) \\
& =\left(\begin{array}{c}
2 r\left(r\left(x+k r(y)-\frac{k}{2}\right)-r\left(y+x+k r(y)-\frac{k}{2}\right)\right)-1 \\
1-2 r\left(y+x+k r(y)-\frac{k}{2}\right)
\end{array}\right) \\
& =\left(\begin{array}{c}
2 r\left(x+k r(y)-\frac{k}{2}-y-x-k r(y)+\frac{k}{2}\right)-1 \\
2 r\left(-y-x-k r(y)+\frac{k}{2}\right)-1
\end{array}\right) \\
& =\left(\begin{array}{c}
2 r\left(-y-x-k r(y)+\frac{k}{2}\right)-1
\end{array}\right) \\
& =\left(\begin{array}{c}
2 r\left(-\frac{-v+1}{2}-r\left(\frac{u-v}{2}\right)-k r\left(\frac{-v+1}{2}\right)+\frac{k}{2}\right)-1
\end{array}\right) \\
& =\left(\begin{array}{c}
2 r\left(\frac{-u+2 v+1}{2}-k \frac{-v+1}{2}+\frac{k}{2}\right)-1
\end{array}\right) \\
v & \left(\begin{array}{c}
2 r\left(\frac{-u+(2+k) v+1}{2}\right)-1
\end{array}\right) .
\end{aligned}
$$


If we set $a=2+k$ then this is precisely the map (5), therefore the two systems are conjugate everywhere except possibly on their zero measure set of discontinuities. The eigenvalues of constant Jacobian of the maps are on the unit circle at $e^{ \pm i \theta}$ where $a=2+k=2 \cos \theta$. 


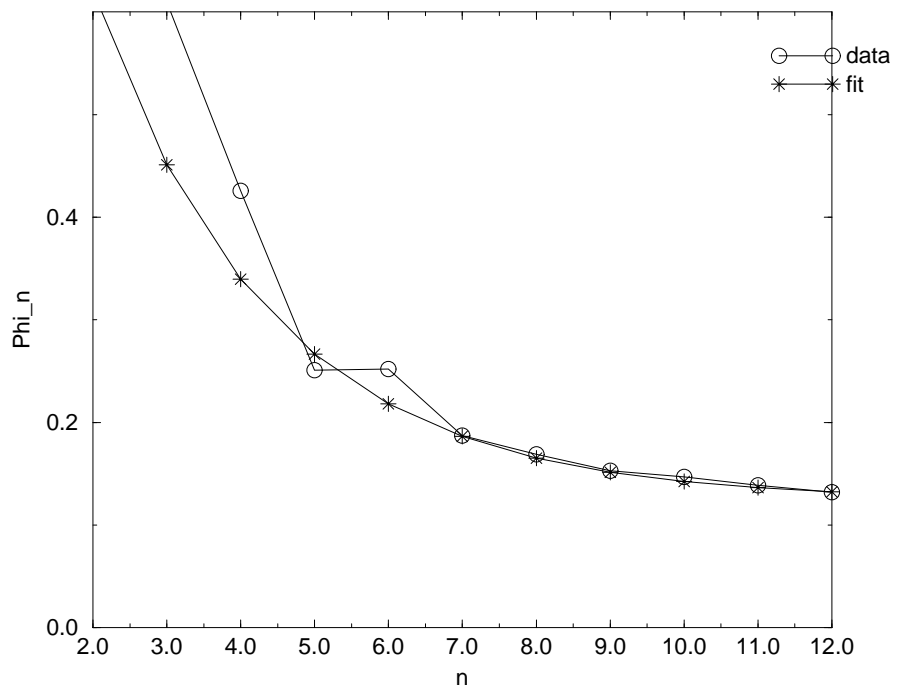

Figure 1: Graph of $\Phi_{n}$ as a function of $n$ for $k=-0.5, \theta=\cos ^{-1} 0.75$. Observe the fit to $\Phi_{n} \sim 0.121+B e^{C n}$ consistent with $\Phi(\theta)=P(\theta)=0.121$ approximately. 


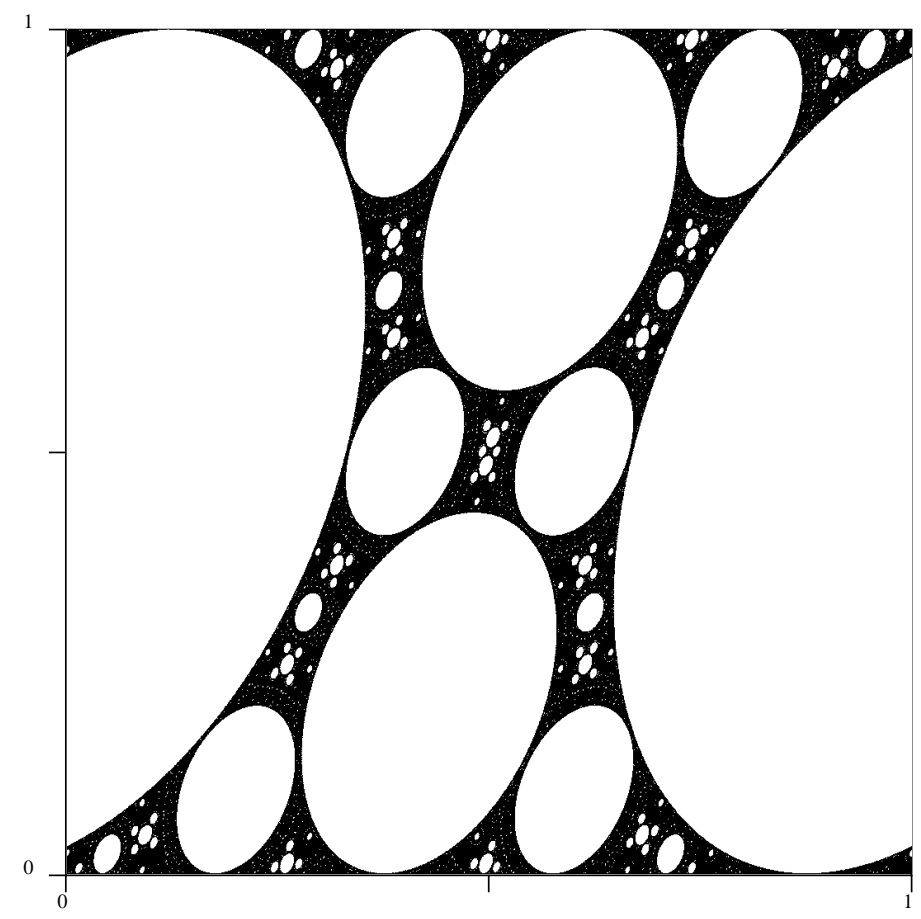

(a)

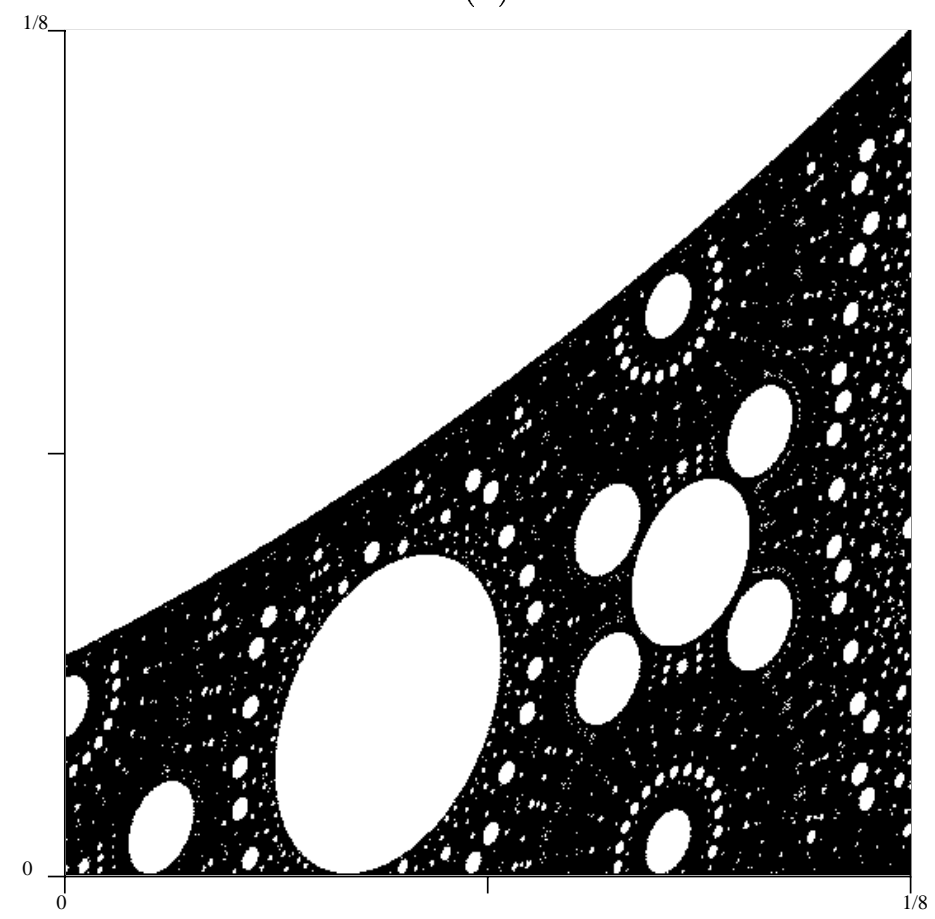

(b)

Figure 2: The area in black is an approximation of $D$ for $k=-0.5$; (a) shows the approximation using $n=10$ in the square $[0,1]^{2}$, (b) shows a detail of the bottom left corner $\left[0, \frac{1}{8}\right]^{2}$ using $n=14$. Note the lack of any exact self-similarity and the presence of increasing proportion of black on examining details of the figure, consistent with $D$ having positive measure. 


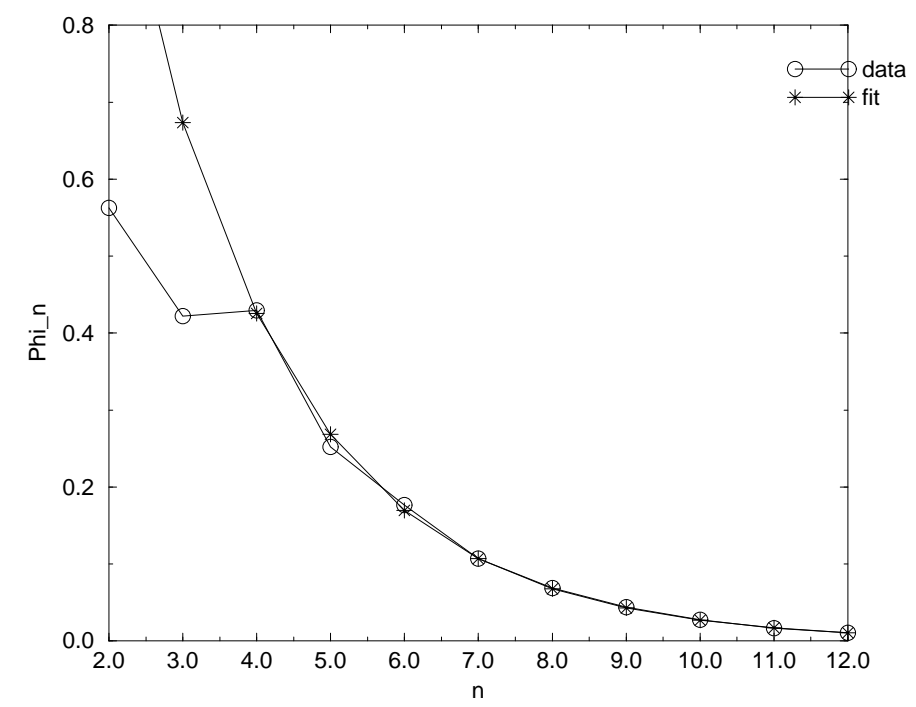

(a)

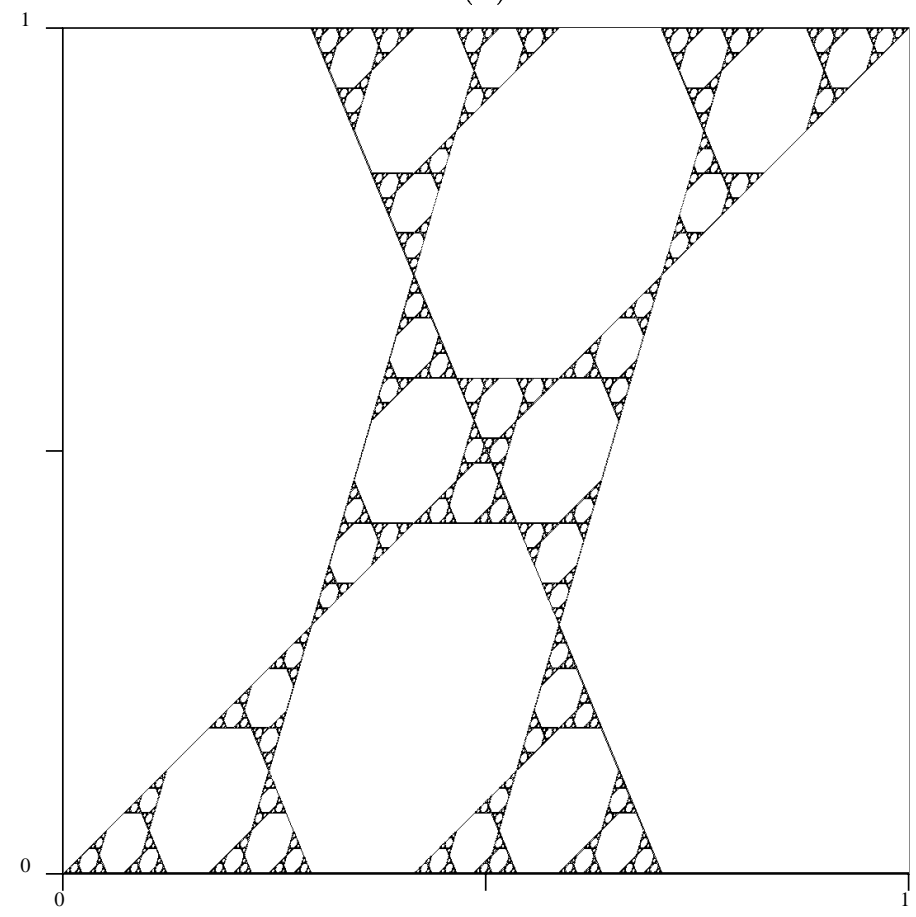

(b)

Figure 3: (a) Graph of $\Phi_{n}$ as a function of $n$ for $k=-2+\sqrt{2}, \theta=\pi / 4$. Observe the fit to $\Phi_{n} \sim B e^{C n}$ consistent with $\Phi(\theta)=P(\theta)=0$. (b) Illustration of the approximation to $D$ for $n=10$. This has a self-similar fractal structure consistent with zero Lebesgue measure and Hausdorff dimension strictly less than two. 


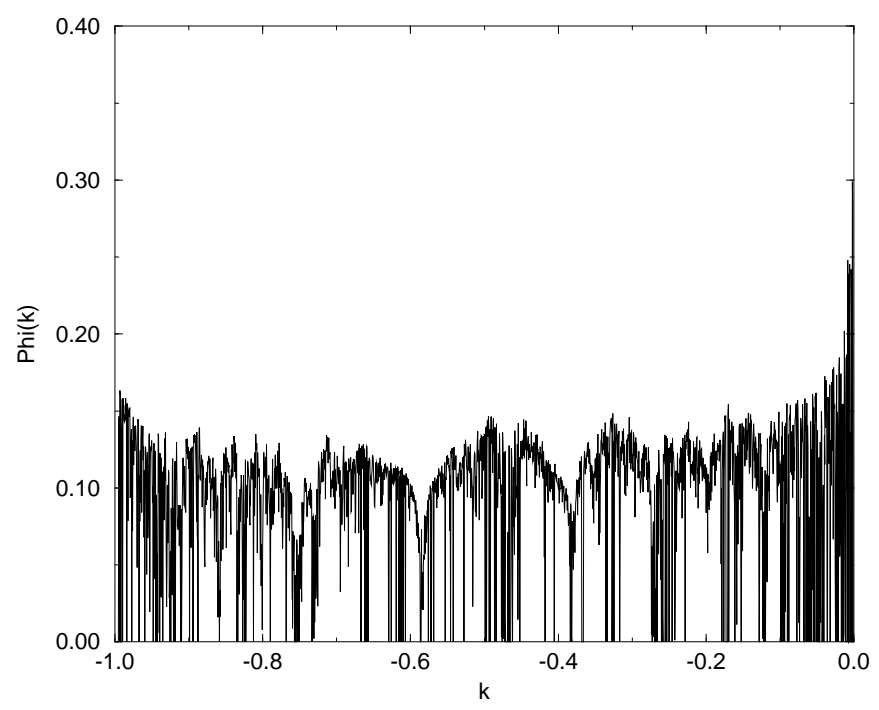

(a)

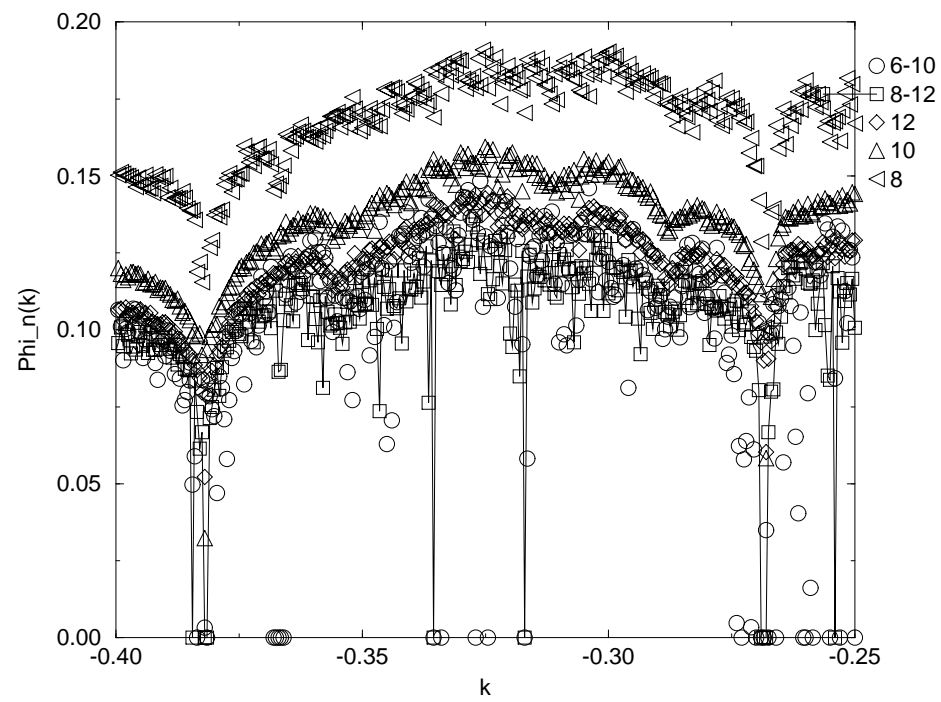

(b)

Figure 4: (a) Approximation of $\Phi_{\infty}$ as a function of $k \in(-1,0)$ made by fitting $\Phi_{n}$ for $n=6,8,10$ to a function $A+B e^{C n}$ and extrapolating to $n=\infty$. (b) Illustration of the convergence of the approximations with $n=6,8,10$ and 12 and the extrapolations using $6-10$ and $8-12$. A small range of $k$ is shown. Note that the fit is uniquely defined if we use only three data points. There is evidence consistent with the extrapolated curve following a continuous curve at irrational values of $\theta / \pi$. 


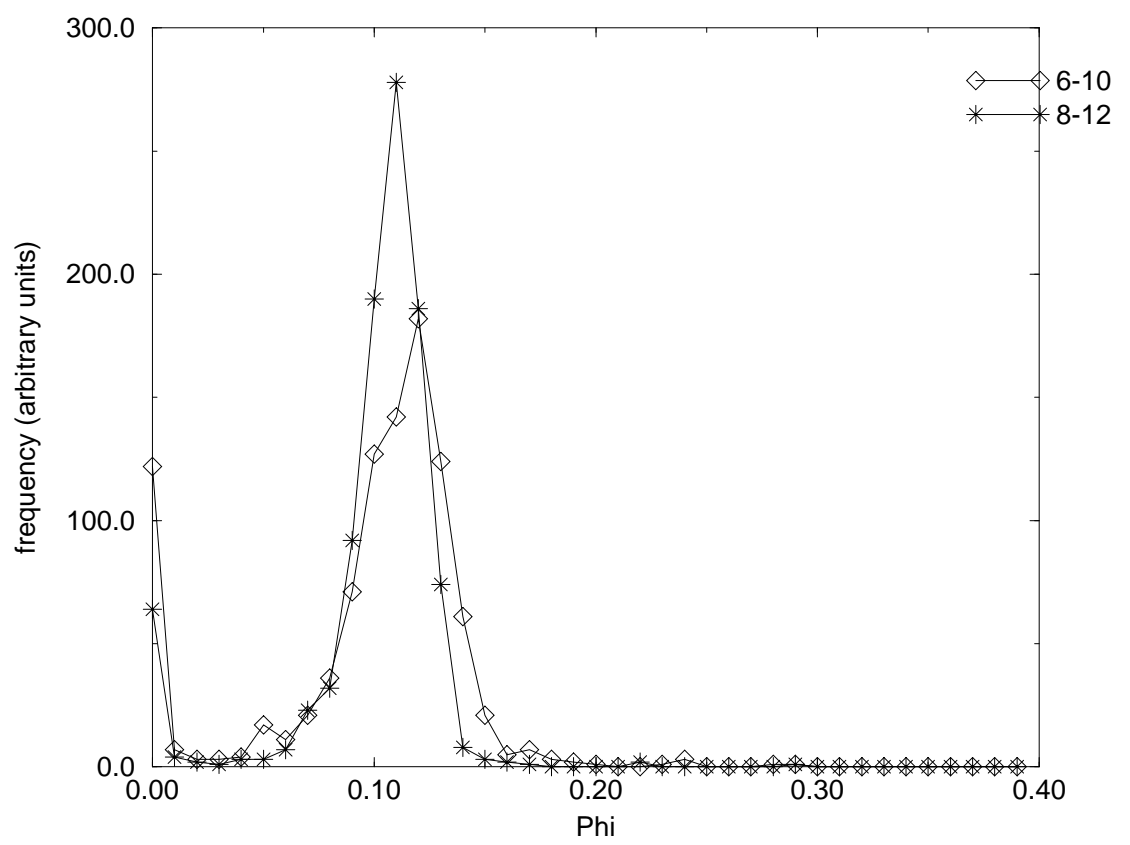

Figure 5: A comparison of the distribution of extrapolated $\Phi_{\infty}$ for $k$ in the range $(-0.5,0)$ calculated using the approximations $n=6-10$ and $n=8-12$. Any spurious points are assigned $\Phi_{\infty}=0$. Note that the latter data reassuringly finds fewer points that are spurious. The mean value of $\Phi$ on this interval of $k$ appears to be of the order of 0.1 . 